\title{
Hierarchical Semantic Labeling for Task-Relevant RGB-D Perception
}

\author{
Chenxia Wu, Ian Lenz and Ashutosh Saxena \\ Department of Computer Science, Cornell University, USA. \\ Email: $\{$ chenxiawu, ianlenz, asaxena\}ecs.cornell.edu
}

\begin{abstract}
Semantic labeling of RGB-D scenes is very important in enabling robots to perform mobile manipulation tasks, but different tasks may require entirely different sets of labels. For example, when navigating to an object, we may need only a single label denoting its class, but to manipulate it, we might need to identify individual parts. In this work, we present an algorithm that produces hierarchical labelings of a scene, following is-part-of and is-type-of relationships. Our model is based on a Conditional Random Field that relates pixel-wise and pair-wise observations to labels. We encode hierarchical labeling constraints into the model while keeping inference tractable. Our model thus predicts different specificities in labeling based on its confidence-if it is not sure whether an object is Pepsi or Sprite, it will predict soda rather than making an arbitrary choice. In extensive experiments, both offline on standard datasets as well as in online robotic experiments, we show that our model outperforms other stateof-the-art methods in labeling performance as well as in success rate for robotic tasks.
\end{abstract}

\section{INTRODUCTION}

Semantic scene labeling is crucial to many robotic tasks, allowing a robot to precisely localize objects, build maps, perform mobile manipulation tasks, and achieve many other goals. In recent work, many algorithms have been developed to produce such a labeling for RGB-D images (e.g., [37, 24, 4, 14]). However, these approaches produce only a flat labeling of a scene, ignoring important relationships between the label classes. In this work, we present an algorithm whose output is a hierarchical labeling of the scene.

These hierarchical labels are very important for a wide range of robotic applications. Segmenting object parts, such as handles, knobs, and buttons, separately from the body of the object is critical to properly afford most household objects. Understanding hierarchical object classes can also enable a robot to make rational substitutions between objects. Consider, for example, the task of fetching a Coke from a fridge (Fig. 1). To open the fridge, the robot must detect and grasp the fridge handle separately from its door. Then, if a Coke is not present in the fridge, it is much more desirable for the robot to return with another soda, such as a Pepsi, than empty-handed.

Using a semantic label hierarchy as shown in Fig. 2 enables these behaviors, which could not be realized using flat labels. When labeling with this hierarchy, each pixel belongs to a series of increasingly-general labels - for example, a pixel of class fridge-handle would also be of classes fridge-door, fridge and electronics. This also allows us to represent uncertainty, using a more general class when the algorithm is not sure which low-level class a pixel should belong to.

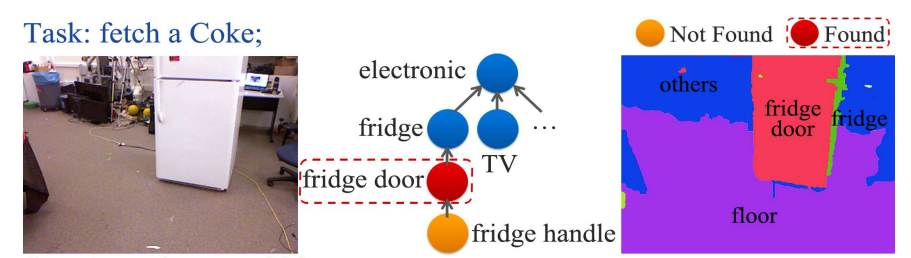

Step 1: navigate to fridge;

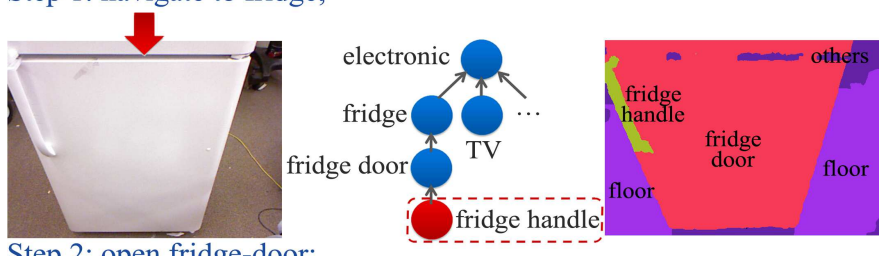

Step 2: open fridge-door;
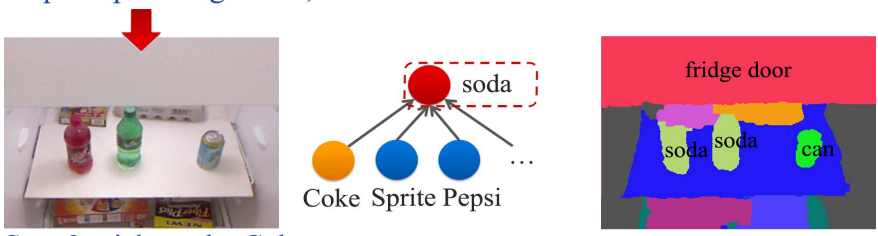

Step 3: pick up the Coke.

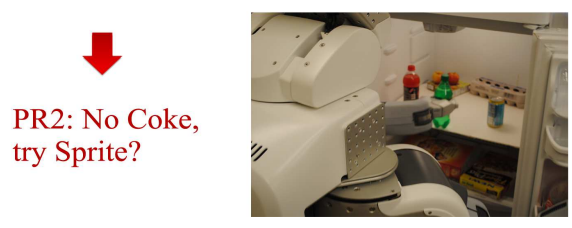

Fig. 1: Hierarchical Labels are produced by our algorithm as required for a robotic task. In the above environment, a robot is asked to fetch a Coke. It needs to perform three sub-tasks: navigate to the fridge, open the fridge door, and pick up the Coke (shown in three rows). For navigation, the robot needs to produce a higherlevel fridge-door label so that it can approximately navigate close to it. Once it gets closer, producing a more detailed fridge-handle label is necessary. In the last step, the robot cannot detect Coke, so it fetches another soda instead. Such a label hierarchy lets a robot hedge its bets.

Conventional flat labeling approaches [37, 14] might simply be applied by flattening all the classes in the semantic hierarchy, but this sacrifices important information. Meanwhile, image classification approaches using semantic hierarchies [10, 35], which predict only one label for each image, cannot be applied to most robotic tasks that require pixel-level labeling of the entire scene. Properly integrating a semantic hierarchy into the labeling problem is a major challenge, and the main focus of this work.

To this end, we propose a novel approach which uses mixed integer programming to optimize a model isomorphic to a Conditional Random Field (CRF). Our model encodes 


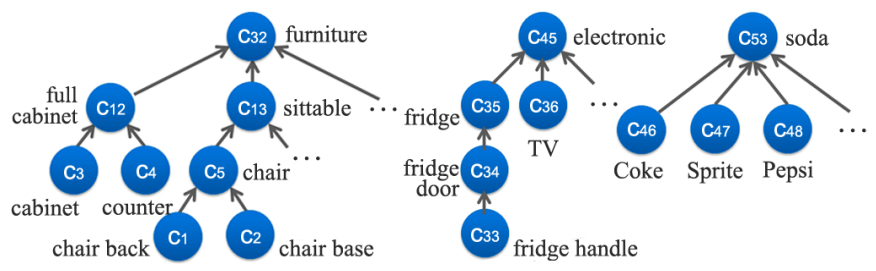

Fig. 2: Semantic hierarchy graph. Each node denotes a class and each directed edge denotes a 'belong to' relation.

relations both from color/depth features to labels and between neighboring segments, as well as constraints arising due to the hierarchical nature of labels. It directly integrates hierarchical information, allowing it to represent ambiguities in perception by giving more general labels. In fact, our algorithm allows a desired specificity of the produced labels, allowing for more specific ones for tasks which need them, and more general ones for those that do not. Our approach also combines multiple segmentation trees generated using different metrics to yield more robust labeling results. We demonstrate that all the necessary terms and constraints for our approach can be combined into a model which remains parsimonious and solvable in under 1.5 seconds per image despite incorporating more information than considered in other labeling algorithms.

We validate the performance of our algorithm in an extensive series of experiments, both offline on the NYUD2 dataset [45] and online in a series of robotic experiments using our PR2 robot equipped with a Microsoft Kinect. Our algorithm produces significantly improved results on hierarchical labeling over the state-of-the-art, increasing performance by up to $15 \%$. In robotic experiments, we demonstrate the usefulness of hierarchical as opposed to flat labeling and show that our algorithm can be applied to real-world robotic scenarios, achieving an average success rate of $81 \%$ over several challenging tasks. Video of some of these is available at http: //pr.cs.cornell.edu/sceneunderstanding/

In summary, the main contributions of this work are:

- We consider a hierarchy of semantic labels when labeling RGB-D scenes, which allows the robot to predict taskrelevant labels.

- We design an inference model that incorporates, over a CRF, relations between segment-features and labels, relations between neighboring segments, as well as constraints arising because of the hierarchical nature of the labels. We show that it still remains tractable and is solved by constrained mixed integer programming.

- Our model allows a robot to choose varying levels of specificity in the labels produced.

- We perform extensive evaluation on the NYUD2 dataset as well as on several different robotic tasks.

\section{RELATED WORK}

Scene understanding. Scene understanding from 2D images has been widely explored [41, 43, 12, 8]. Due to the availability of affordable RGB-D sensors, significant effort has been put into RGB-D scene understanding recently [45, 37. 24, 31, 4, 25, 14, 20, 19, 17, 27]. Ren et al. [37] developed
Kernel Descriptors, highly useful RGB-D feature, and used the segmentation tree to get contextual information. Gupta et al. [14] generalized $2 \mathrm{D} \mathrm{gPb}-\mathrm{ucm}$ contour detection to $3 \mathrm{D}$, giving more effective segmentation. Koppula et al. [24] and Anand et al. [4] used rich contextual information for semantic labeling of 3D point clouds. Jia et al. [19] interpreted objects in a scene by reasoning about blocks, support, and stability. All these works predict flat labels, which are not applicable to many robotic tasks. Instead, our approach outputs a hierarchical labeling, which aids navigation, object finding and rational target substitution in robotic applications.

Visual recognition using semantic hierarchies. Our work is also related to visual recognition using semantic hierarchies [9, 39]. One similar work [10] classified large scale images by optimizing accuracy-specificity trade-offs. Ordonez et al. [35] considered predicting labels that people actually use to name an object. Both of these works targeted web image classification, and so predict a single label for each image denoting the most salient object. For many robotic tasks, we must consider pixel level labeling of multiple objects in a complex scene using a semantic hierarchy.

Robotic tasks using vision. There is also a huge body of works using vision algorithms to help perform different robotic tasks [13, 38, 16, 30], such as object grasping [42, 11, 29], navigation [6, 26], trajectory control [44], and activity anticipation [23]. Many works focused on improving SLAM techniques to better depict an environment for planning and navigation [34, 28], such as incremental smoothing and mapping using the Bayes Tree [21], real-time visual SLAM over large-scale environments [46], and object level SLAM [40]. Milford [33], He and Upcroft [15] proposed a place recognition algorithm for mobile robots. Katz and Brock [22] developed interactive segmentation for observing object motion during manipulation. Pangercic et al. [36] built semantic object maps for manipulation tasks for an autonomous service robot. Hinkle and Edwin [18] proposed a technique for functionally classifying objects using features obtained through physical simulations.

\section{OVERVIEW}

The input to our algorithm is a co-registered RGB and Depth image pair $I \in \mathbb{R}^{m \times n \times 3}, D \in \mathbb{R}^{m \times n}$, where $m, n$ are the image height and width. Our goal is to predict the label of each pixel and output the label matrix $L \in C^{m \times n}$, where $C$ is the set of possible hierarchical semantic labels. We achieve this by mapping a semantic hierarchy graph to the segmentation tree built on the input image. We will first introduce the semantic hierarchy graph and the segmentation tree in this section.

Semantic hierarchy graph. For many robotic actions, we need semantic labels at different levels of abstraction rather than a simple object level. Therefore, we consider two types of relations in a semantic hierarchy:

- Is-part-of. For some robotic tasks, we need detailed localization of specific object parts. For example, to open a fridge, it is much better to know exactly where the fridge-handle 
is from the labeling rather than to simply guess based on a higher-level fridge-door label.

- Is-type-of. Understanding which objects belong to the same higher-level semantic class allows a robot to make rational substitutions between such objects. For example, if the robot is sent to find a Coke but cannot, it could instead return with any soda such as a Pepsi.

We represent this semantic hierarchy by a directed acyclic graph, called a semantic hierarchy graph, where the nodes $C=\left\{c_{k}\right\}$ represent the possible labels and the edges represent one of aforementioned relations. See Fig. 2 for an example.

Segmentation tree of the RGB-D image. We begin by segmenting the image into small segments. This gives us a set of candidate segments $\left\{s_{i}\right\}$ to label. If a segment is too small, visual and/or geometric information might be limited; if it is too large, it might straddle a class boundary. We therefore build a segmentation tree and label over this tree. In detail, we first obtain leaf node over-segmentations using a $\mathrm{gPb}$ ucm approach extended for RGB-D images [14]. Second, we merge the most similar pairs of nodes step-by-step based on a similarity measure (the $\mathrm{gPb}$-ucm boundary value 1 , forming a tree as shown in Fig. 3 .

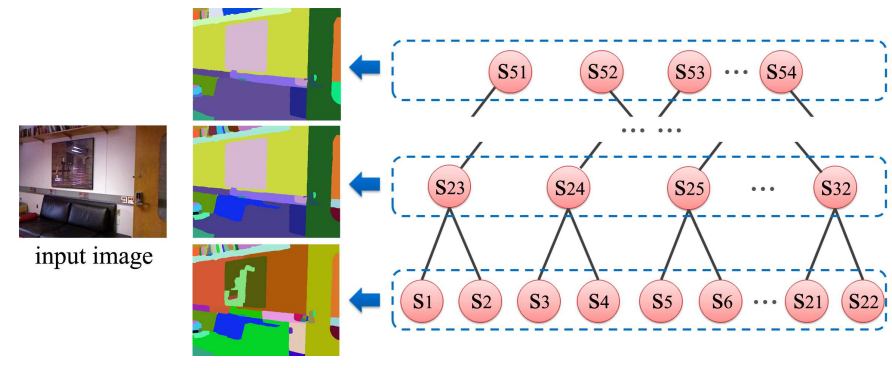

Fig. 3: Illustration of segmentation tree. Pixels are grouped into small segments which are then merged to form a segmentation tree.

Note that mapping the semantic hierarchy graph to the segmentation tree is challenging, because both labels and segments are hierarchical rather than flat as in previous works. For example, for a parent segment with two child segments, it is possible to label them with parent-child labels such as labeling the parent as chair and the children as chair-back and chair-base, or to only label the children as two unrelated classes such as $T V$ and cabinet. Thus, we need to take into account appropriate constraints in designing our CRF-based objective function. For many robotic applications, it is also desirable to be able to select the degree of specificity of the produced labels in the semantic hierarchy. Integrating all these desiderata into a parsimonious model is challenging.

\section{Preliminaries}

Our approach is based on a Conditional Random Field (CRF), modeling the unary terms of, and pair-wise relations

\footnotetext{
${ }^{1}$ In order to improve the chances of obtaining desirably-sized segments for labeling, we actually build multiple segmentation trees based on different similarity measures [32]: the $\mathrm{gPb}-\mathrm{ucm}$ boundary value ( $\mathrm{ucm}$ tree), the similarities between the normals of two neighboring segments (normal tree), and the semantic similarities of any two segments (category tree). These diverse trees provide rich candidate segments for the labeling stage.
}

between, the segments. We will introduce the unary term and a CRF model to label RGB-D images with flat-labels in this section. We first define the following notations:

$c_{k} \quad k$-th label in the semantic hierarchy graph.

$s_{i} \quad i$-th segment in the segmentation tree.

$y_{i k} \in\{0,1\}$. If $s_{i}$ is labeled with $c_{k}, y_{i k}=1$,o.w. $y_{i k}=0$.

$a_{i}$ number of pixels in segment $s_{i}$.

$a_{i k}$ number of pixels of class $c_{k}$ in segment $s_{i}$.

$w_{i k}=a_{i k} / a_{i}$, fraction of $c_{k}$ class pixels in segment $s_{i}$.

\section{A. Unary term of a segment}

The unary term relates the features of a segment to its label. Kernel descriptors have been proven to be useful features for RGB-D scene labeling [37], so we extract six such descriptors from each segment: gradient, color, local binary pattern, depth gradient, spin, surface normals, and KPCA/self-similarity. The feature vector of segment $s_{i}$ is denoted as $\mathbf{z}_{i}$. We then use the fraction of $c_{k}$ class pixels in segment : $w_{i k}^{*}=a_{i k} / a_{i}$ as a confidence score for $s_{i}$ belonging to $c_{k}$. Since each pixel belongs to several ground-truth classes in the hierarchy such as chair-back, chair, sittable, furniture, we treat this as a linear regression problem rather than a classification problem as in previous work [37]. In detail, ridge linear regression is used to train the linear prediction function $\hat{w}_{i k}=\theta_{k}^{\top} \mathbf{z}_{i}$.

\section{B. Labeling RGB-D Images with Flat Labels}

Previous work by Anand et al. [4] started by dividing the RGB-D image into small segments, with the goal of labeling each segment from a flat label set $\left\{c_{k}\right\}$. They then used a CRF to model the unary terms of and pair-wise relations between the segments. Since each segment is allowed to belong to only one class, we have the constraint $\sum_{c_{k}} y_{i k}=1$. The objective function is as follows:

$$
\begin{aligned}
& \max _{\mathbf{y}} \overbrace{\sum_{s_{i}, c_{k}} y_{i k} \hat{w}_{i k}}^{\text {FlatSeg-FlatLabel } \mathbf{y}}+\overbrace{\sum_{\left(s_{i}, s_{j}\right) \in \mathbb{N}, c_{k}} y_{i k} y_{j k} \Phi\left(s_{i}, s_{j}\right)}^{\text {unary terms }}, \\
& \text { s.t. } \quad \sum_{c_{k}} y_{i k}=1 \quad \forall s_{i}, \quad y_{i k} \in\{0,1\} .
\end{aligned}
$$

Here the unary term is $\hat{w}_{i k}$, the edge term is $\Phi\left(s_{i}, s_{j}\right)=$ $\alpha \exp \left(-\beta g P b\left(s_{i}, s_{j}\right)\right)$, in which $g P b\left(s_{i}, s_{j}\right)$ is the gPb-ucm boundary weight between $s_{i}, s_{j}$, and $\alpha, \beta$ are two weighting parameters. The edge term encourages neighboring segments $\left(s_{i}, s_{j}\right) \in \mathbb{N}$ with small boundaries to take the same label.

\section{OUR APPROACH}

In this section, we will describe an improved CRF model with constraints which allow labeling over semantic trees using hierarchical labels. We first define the following notations:

$\pi(v)$ a function that takes a vertex $v$ in a directed graph and returns the set of its ancestors, including itself.

$\dot{\pi}(v)$ the set of ancestors without $v$ itself: $\pi(v)-\{v\}$.

$\check{s}_{l} \quad l$-th leaf node segment in the segmentation tree.

$H_{l}$ hierarchical relation graph of the ancestor set $\pi\left(\check{s}_{l}\right)$.

$\mathbb{Q}_{l t} \quad t$-th maximal independent set of graph $H_{l}$. 


\section{A. Labeling Segmentation Trees with Flat Labels}

Now we describe how we label a segmentation tree, where flat segments are merged to form a tree as in Fig. 3 As some segments in the tree overlap, we first need to select which ones to label, and second predict their labels. We achieve this by enforcing that, for each leaf node segment, only one of its ancestors (including itself) is labeled. This is because a pixel can have only one label in a flat labeling scheme while these segments are overlapping. So following constraints are added.

Non-overlapping constraints (NO-CT). We replace the sum-to-one constraint $\sum_{c_{k}} y_{i k}=1, \forall s_{i}$ in Eq. 1 with $\sum_{s_{i} \in \pi\left(\check{s}_{l}\right), c_{k}} y_{i k}=1, \forall \check{s}_{l}$. Since all leaf nodes are considered, every pixel is labeled with exactly one label. We also need to ensure that the area of the child segment vs. the parent segment is accounted for in the objective function. We therefore weight each $\hat{w}_{i k}$ by the total number of pixels $a_{i}$ of the segment $s_{i}$. The objective function then becomes:

$$
\begin{aligned}
& \text { TreeSeg-FlatLabel }_{\mathbf{y}}(\hat{\mathbf{w}}, \mathbf{a}, \Phi) \text { : } \\
& \max _{\mathbf{y}} \overbrace{\sum_{s_{i}, c_{k}} y_{i k} \hat{w}_{i k} a_{i}}^{\text {unary terms }}+\overbrace{\sum_{\left(s_{i}, s_{j}\right) \in \mathbb{N}, c_{k}} y_{i k} y_{j k} \Phi\left(s_{i}, s_{j}\right)}^{\text {edge terms }}, \\
& \text { s.t. } \overbrace{\sum_{s_{i} \in \pi\left(\check{s}_{l}\right), c_{k}} y_{i k}=1 \quad \forall \check{s}_{l}}^{\text {NO-CT }}, \quad y_{i k} \in\{0,1\} \text {. }
\end{aligned}
$$

\section{B. Labeling Segmentation Trees with Hierarchical Labels}

When hierarchical labels are introduced, the following interesting property emerges: even if a child node is labeled, its ancestors can be labeled with its ancestor classes. This complicates the specification of constraints in the model, so we add following hierarchical relation constraints. We summarize our RGB-D hierarchical semantic labeling approach in Alg. 1.

Hierarchical relation constraints (HR-CT). Now, we allow labeling more than one segment in $\pi\left(\check{s}_{l}\right)$ with hierarchical labels, such as labeling the parent node as chair and the child as chair-back. To achieve this, we do the following:

1) Find hierarchical relations. We first define a tuple $\left(s_{i}, s_{j}, c_{k}, c_{z}\right)$, called hierarchical relation if it follows $c_{z} \in \dot{\pi}\left(c_{k}\right), s_{j} \in \dot{\pi}\left(s_{i}\right)$. This allows the pair of segments $\left(s_{i}, s_{j}\right) \in \pi\left(\breve{s}_{l}\right)$ to be labeled with $\left(c_{k}, c_{z}\right)$ respectively, as their order is consistent in both the segmentation tree and the semantic hierarchy graph. All such tuples comprise a set $\Omega_{l}$ for each $\pi\left(\check{s}_{l}\right)$.

2) Build hierarchical relation graph. In order to find all the constraints in each ancestor-set $\pi\left(\check{s}_{l}\right)$ considering both the non-overlapping and hierarchical labeling properties, we build a undirected graph $H_{l}=\left(\mathbb{V}_{l}, \mathbb{E}_{l}\right)$, called $h i$ erarchical relation graph, of which the vertices are all possible assignments: $\mathbb{V}_{l}=\left\{y_{i k}, \forall s_{i} \in \pi\left(\check{s}_{l}\right), \forall c_{k}\right\}$ and edges link vertices if they follow the hierarchical relation: $\mathbb{E}_{l}=\left\{\left(y_{i k}, y_{j z}\right), \forall\left(s_{i}, s_{j}, c_{k}, c_{z}\right) \in \Omega_{l}\right\}$.

3) Find constraints on the hierarchical relation graph. Following the hierarchical relation, if two vertices $\left(y_{i k}, y_{j z}\right)$ on $H_{l}$ are linked by an edge, they can be both set to one.

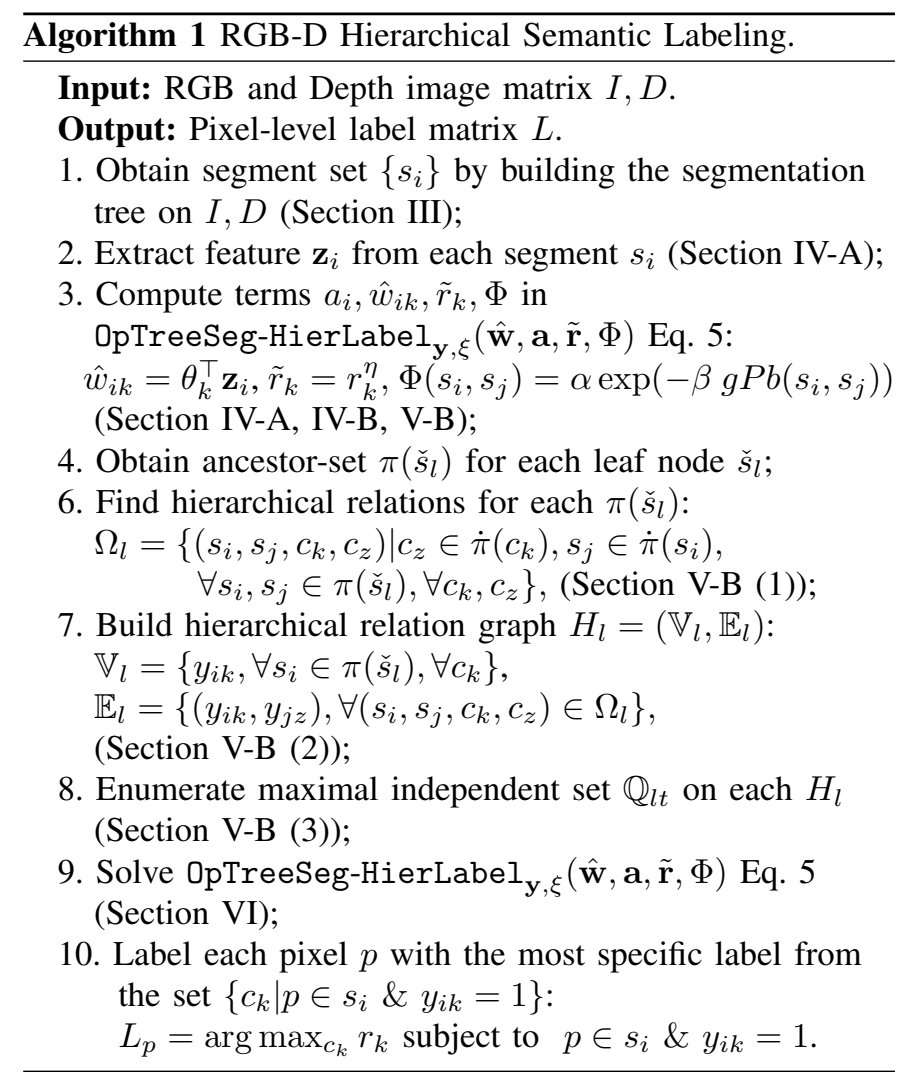

Otherwise, at most one can be set to one following the non-overlapping constraint. To give efficient and sufficient constraints, we constrain the sum of all $y_{i k}$ in each maximal independent set (the set of vertices, no pair of which are adjacent) to be not greater than one. The problem then becomes to enumerate all maximal independent set ${ }^{2}$ $\left\{\mathbb{Q}_{l t}, t=1, \ldots\right\}$ of $H_{l}$. In practice, we will introduce a parsimonious model (Sec. VI), leading to a sparse graph $H_{l}$ thus more efficient constraint-finding. After finding these sets, we add the constraints $\sum_{y_{i k} \in \mathbb{Q}_{l t}} y_{i k} \leq 1, \forall c_{l}, t$. To further ensure that all pixels to be labeled, we add the completeness constraints (CM-CT) $\sum_{s_{i} \in \pi\left(\breve{s}_{l}\right), c_{k}} y_{i k} \geq 1, \forall l$ to ensure at least one segment in each $\pi\left(\check{s}_{l}\right)$ to be labeled.

4) Overlapping unary correction. To give even weighting for each pixel, we also modify the unary term for the overlapping pixels when both parent and child segments are labeled. If $y_{i k}$ and $y_{j z}$ are both set to $1, y_{i k} y_{j z}=1$, when $\left(s_{i}, s_{j}, c_{k}, c_{z}\right) \in \Omega_{l}$, we would rather label their overlapping pixels $a_{i}$ with the more specific label $c_{k}$. So, the summation of the unary term would be $\hat{w}_{i k} a_{i}+\hat{w}_{j z}\left(a_{j}-a_{i}\right)$. Then, the objective function relating these two terms changes to $y_{i k} \hat{w}_{i k} a_{i}+y_{j z} \hat{w}_{j z} a_{j}-y_{i k} y_{j z} \hat{w}_{j z} a_{i}$.

Note that considering these hierarchical relations and con-

\footnotetext{
${ }^{2}$ To enumerate maximal independent sets of $H_{l}$, we first divide $H_{l}$ into a subgraph $\left(\tilde{\mathbb{V}}_{l}, \emptyset\right)$, where $\tilde{\mathbb{V}}_{l}$ are all isolated vertices in $H_{l}, \emptyset$ is the empty edge set, and another subgraph $\tilde{H}_{l}=\left(\mathbb{V}_{l}-\tilde{\mathbb{V}}_{l}, \mathbb{E}_{l}\right)$. Then we enumerate all maximal independent sets $\left\{\tilde{\mathbb{Q}}_{l t}, t=1, \cdots\right\}$ of $\tilde{H}_{l}$ by enumerating all cliques of its complementary graph, which is a well-studied problem in graph theory [2] 7] and is solved by the Bron-Kerbosch algorithm [7] in our approach. Finally, $\mathbb{Q}_{l t}=\tilde{\mathbb{Q}}_{l t} \cup \tilde{\mathbb{V}}_{l}$.
} 

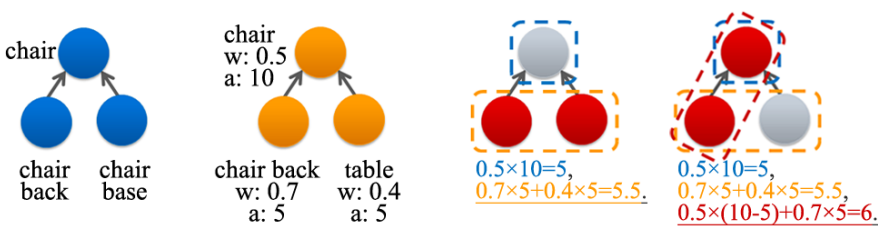

(a) Ground truth. (b) Estimated score.

(c) NO-CT.

(d) NO-CT, HR-CT.

Fig. 4: An illustration of the benefit of adding HR-CT. In the example, (a) shows the ground-truth labels of the segments. (b) gives the highest estimated confidence score $\hat{w}$, its corresponding estimated label and the area $a$ of each node. (c) considers nonoverlapping segments selection leading to two possible selections and (d) further considers the hierarchical relation leading to one more possible selection. According to the sum of scores, (c) fails to label the right child node while (d) gives a reasonable labeling, because the (chair,chair-back) relation strengthens each other avoiding the possible error incurred by the poor estimated $\hat{w}$.

straints allows the model to avoid possible errors caused by poor local estimates of $\hat{w}$ (see an example in Fig. 4).

Choosing the degree of specificity for hierarchical labels. For many robotic applications, it is also desirable to be able to decide the degree of specificity of the produced labels. Here we use the information gain $r_{k}$ to represent the specificity of each class as in [10]:

$$
r_{k}=\log _{2}|C|-\log _{2} \sum_{c_{z} \in C} I\left(c_{k} \in \pi\left(c_{z}\right)\right),
$$

where the first term is the total number of classes and the second term gives the number of $c_{k}$ 's child nodes. We can see $r_{k}$ is larger for lower level classes and smaller for higher levels in the semantic hierarchy. We weight the unary term $\hat{w}_{i k}$ by $\tilde{r}_{k}=r_{k}^{\eta}$, where $\eta$ is the parameter deciding the degree of specificity of prediction ${ }^{3}$

In summary, the final objective function becomes:

$$
\begin{aligned}
& \text { TreeSeg-HierLabel } \mathbf{y}(\hat{\mathbf{w}}, \mathbf{a}, \tilde{\mathbf{r}}, \Phi) \text { : } \\
& \max _{\mathbf{y}} \overbrace{\sum_{s_{i}, c_{k}} y_{i k} \hat{w}_{i k} \tilde{r}_{k} a_{i}}^{\text {unary }}-\overbrace{\sum_{\check{s}_{l},\left(s_{i}, s_{j}, c_{k}, c_{z}\right) \in \Omega_{l}} y_{i k} y_{j z} \hat{w}_{j z} \tilde{r}_{z} a_{i}}^{\text {terms }} \\
& +\overbrace{\sum_{\left(s_{i}, s_{j}\right) \in \mathbb{N}, c_{k}} y_{i k} y_{j k} \Phi\left(s_{i}, s_{j}\right)}^{\text {edge terms }} \\
& \text { s.t. } \overbrace{\sum_{y_{i k} \in \mathbb{Q}_{l t}} y_{i k} \leq 1 \forall \check{s}_{l}, t}^{\mathrm{NO}-\mathrm{CT}, \mathrm{HR}-\mathrm{CT}}, \overbrace{\sum_{\sum_{i} \in \pi\left(\check{s}_{l}\right), c_{k}} y_{i k} \geq 1 \forall \check{s}_{l}}^{\mathrm{CM}-\mathrm{CT}} \text {, } \\
& y_{i k} \in\{0,1\} \text {. }
\end{aligned}
$$

After solving this, we label each pixel $p$ with the most specific label from the set: $\left\{c_{k} \mid p \in s_{i} \& y_{i k}=1\right\}$.

\section{EFFICIENT OPTIMIZATION}

The quadratic term in the objective function makes optimization difficult. So, we equivalently formulate it by replacing quadratic term $y_{i k} y_{j z}$ with an auxiliary variable $\xi_{i j}^{k z}$

\footnotetext{
${ }^{3}$ With larger $\eta$, the relative weight for more specific class: $\left(r_{i} / r_{j}\right)^{\eta}, r_{i}>$ $r_{j}$ is larger, thus prediction is more specific. The prediction is balanced when $\eta=0$.
}

leading to a linear objective which can be solved by a mixed integer programming (MIP) solver [1]:

$$
\begin{aligned}
& \text { OpTreeSeg-HierLabel } \mathbf{y}_{, \xi}(\hat{\mathbf{w}}, \mathbf{a}, \tilde{\mathbf{r}}, \Phi) \text { : } \\
& \max _{\mathbf{y}, \xi} \overbrace{\sum_{s_{i}, c_{k}} y_{i k} \hat{w}_{i k} \tilde{r}_{k} a_{i}}^{\text {unary terms }}-\overbrace{\sum_{\check{s}_{l},\left(s_{i}, s_{j}, c_{k}, c_{z}\right) \in \Omega_{l}} \xi_{i j}^{k z} \hat{w}_{j z} \tilde{r}_{z} a_{i}}^{\text {overlapping correction terms }} \\
& +\overbrace{\sum_{\left(s_{i}, s_{j}\right) \in \mathbb{N}, c_{k}} \xi_{i j}^{k k} \Phi\left(s_{i}, s_{j}\right)}^{\text {edge terms }}, \\
& \text { s.t. } \overbrace{\sum_{y_{i k} \in \mathbb{Q}_{l t}} y_{i k} \leq 1 \forall \check{s}_{l}, t}^{\mathrm{NO}, \mathrm{CT}, \mathrm{HR}-\mathrm{CT}} \overbrace{\sum_{\sum_{i} \in \pi\left(\check{s}_{l}\right), c_{k}} y_{i k} \geq 1 \forall \check{s}_{l}}^{\mathrm{CM}-\mathrm{CT}} \text {, } \\
& \xi_{i j}^{k z} \leq y_{i k}, \xi_{i j}^{k z} \leq y_{j z}, y_{i k}+y_{j z} \leq \xi_{i j}^{k z}+1, \forall s_{i}, c_{k} \\
& y_{i k} \in\{0,1\}, \quad \xi_{i j}^{k z} \in\{0,1\} \text {, }
\end{aligned}
$$

Parsimonious Model. We observe that there is some redundancy in the above objective, and introduce a parsimonious model to avoid this.

First, we do not need to consider all possible classes for each segment. Classes with low unary terms $\hat{w}_{i k} \tilde{r}_{k} a_{i}$ can be omitted for $s_{i}$. We consider only the top $\tau$ classes, leaving only $\tau$ possible $y_{i k}$ for each $s_{i}$.

Second, in constraint-finding, some hierarchical relations $\left(s_{i}, s_{j}, c_{k}, c_{z}\right) \in \Omega_{l}$ are mutually exclusive ${ }^{4}$ So we also consider $\hat{w}_{i k} \tilde{r}_{k} a_{i}$ in each hierarchical relation, reducing the number of relations by greedily selecting the top ones with no conflicts. In detail, we first rank all the possible hierarchical relations $\left(s_{i}, s_{j}, c_{k}, c_{z}\right)$ by the sum of unary terms of each pair $w_{i k} \tilde{r}_{k} a_{i}+w_{j z} \tilde{r}_{z} a_{j}$, all of which consist a candidate relation list. We select the one with the highest score from the list, link the corresponding edge in graph $H_{l}$, and remove all its violating relations from the list. We repeat this selection until no relations remain in the list. As a result, the graph $H_{l}$ becomes sparse with many isolated vertices, since only most confident relations are considered.

The most time consuming step in Alg. 1 is to enumerate the maximal independent sets in step 8 . In the worst case it is $O\left(n_{l} 3^{h_{s} \cdot \tau / 3}\right)$, where $n_{l}$ is the number of leaf nodes of and $h_{s}$ is the height of the segmentation tree, and $\tau$ is the number of top considered classes. Though the worst-case running time is non-polynomial, the Bron-Kerbosch algorithm runs much faster in practice [3]. In our experiments on the NYUD2 dataset, it only takes an average of 0.84 and 0.49 seconds per image respectively to find the constraints and optimize the objective using our parsimonious model.

\footnotetext{
${ }^{4}$ For example, consider $\left(s_{1}, s_{3}, c_{1}, c_{2}\right)$ and $\left(s_{2}, s_{4}, c_{3}, c_{4}\right)$, where $s_{2} \in$ $\dot{\pi}\left(s_{1}\right), s_{3} \in \dot{\pi}\left(s_{2}\right), s_{4} \in \dot{\pi}\left(s_{3}\right), c_{2} \in \dot{\pi}\left(c_{1}\right), c_{3} \in \dot{\pi}\left(c_{2}\right), c_{4} \in \dot{\pi}\left(c_{3}\right)$. They are both belong to hierarchical relations according to the definition. However, they are mutually exclusive because when $y_{1,1}=1, y_{3,2}=1, y_{2,3}$ cannot be 1 as the segment $s_{2}$ is within segments $s_{1}, s_{3}$ while class $c_{3}$ is higher than classes $c_{1}, c_{2}$
} 
TABLE I: Average class recall of each class level on NYUD2 dataset.

\begin{tabular}{c|cccc}
\hline Recall(\%) & class level0 & class level1 & class level2 & class level3 \\
\hline \hline$[37 \mid$ & 24.77 & 30.52 & 36.02 & 41.66 \\
{$[14 \mid+[37]$} & 28.96 & 34.14 & 41.69 & 46.29 \\
Ours(bs+bc) & 30.08 & 36.09 & 45.96 & 51.80 \\
Ours(ts+bc) & 32.78 & 41.38 & 49.26 & 55.48 \\
Ours(ts+hc) & $\mathbf{3 3 . 3 5}$ & $\mathbf{4 4 . 4 6}$ & $\mathbf{5 1 . 7 9}$ & $\mathbf{6 1 . 5 1}$ \\
\hline
\end{tabular}

\section{SCEne LABELING EXPERIMENTS}

Data. We evaluate our approach on a hierarchically-labeled subset of the NYUD2 dataset [45], which consists of RGB-D images from a wide variety of environments. We manually labeled a subset of 500 images using a hierarchy. We used 20 most common object classes and one background class, and additionally labeled 12 object-part classes and generalized 10 higher level classes. In total, we have 43 classes in the semantic hierarchy. We use the standard split of the NYUD2 dataset, giving 269 training images and 231 test images.

Implementation details. In our experiments, we used six RGB-D kernel descriptors to represent segments for both [37] and our approach. We kept the same setting as in [37] to run their approach: first, we ran gPb-ucm algorithm [5] on both the RGB and depth images separately and linearly combine them to get the $\mathrm{gPb}-\mathrm{ucm}$ values, then built one segmentation tree by using different values to threshold these values. To make a fair comparison, we also ran the 3D gPm-ucm [14] algorithm to get the $\mathrm{gPb}-\mathrm{ucm}$ value for both approach [37] and ours. So we denote the approach [37] based on original $\mathrm{gPb}-\mathrm{ucm}$ as [37] and based on 3D gPb-ucm as [14]+[37].

Evaluation metric. We use three metrics for evaluating scene labeling performance: cumulative pixel accuracy, average information gain and average class recall. We label each scene image at the pixel level and consider it correct to label a pixel with its ground truth label or any of its ancestors, e.g., a pixel of class chair-back is also of class chair. If $\hat{L}_{p}$ is a prediction of a pixel label and $L_{i}^{*}$ is its ground truth leaf node label, the cumulative pixel accuracy over the whole dataset is defined as: $\sum_{p} I\left(\hat{L}_{p} \in \pi\left(L_{p}^{*}\right)\right) / n_{p}$, where $I($.$) is an indicator function$ and $n_{p}$ is the number of pixels in the whole dataset, $\pi\left(L_{p}^{*}\right)$ is the set of all possible correct predictions including $L_{p}^{*}$ and all its ancestors in the semantic hierarchy.

With hierarchical labels, an algorithm can always predict the top-level parent classes and get higher performance, e.g., it is easier to label furniture vs table-leg. Therefore, following [10], we evaluate the degree of specificity for prediction. Specifically, we compute the information gain (Eq. 3) of each predicted class as defined earlier and compute the average.

Recall for class $c$ is defined as: $\left(\sum_{p} I\left(\hat{L}_{p} \in \operatorname{ch}(c) \& \hat{L}_{p} \in\right.\right.$ $\left.\left.\pi\left(L_{p}^{*}\right)\right)\right) /\left(\sum_{p} I\left(c \in \pi\left(L_{p}^{*}\right)\right)\right)$, where $\operatorname{ch}(c)$ represent the class set of all $c$ 's children plus $c$ itself in the semantic hierarchy. So, the numerator is the number of correctly predicted pixels for class $c$, and the denominator is the number of pixels with $c$ as ground truth label.
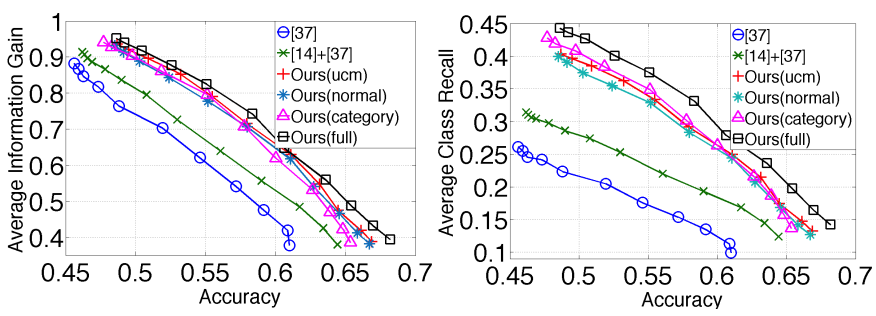

Fig. 5: Results on NYUD2 dataset. For the same degree of specificity for prediction (i.e., same information gain, left) and recall (right), our algorithm performs better.

\section{A. Results}

We first evaluate the average class recall on four levels of the semantic hierarchy. Table. [1 summarizes the results. Class level0 contains the base classes, the most specific classes in the tree, e.g. object parts and low-level object classes. Higher levels are obtained by merging nodes in each previous level, leading to more general classes. Fig. 7 shows all classes for each level.

In this experiment, we train and predict labels on the base classes for flat labeling approaches [37],[14]+[37]. For our approach, we train and predict labels using leaf node segments on the base classes (Ours(ls+bc)), the segmentation tree on the base classes (Ours(ts+bc)) and the segmentation tree on the test class level and all classes below them in the semantic hierarchy (Ours(ts+hc)), with $\eta=0$ for balanced prediction. These results reveal a number of interesting points as follows:

- The proposed approach Ours(ts+hc) shows the best results at each level, even though predicting more hierarchical labels is harder than the task of the other compared approaches, which only predict the base classes. This is because our approach effectively considers the mapping of the semantic hierarchy to the segmentation tree.

- Labeling on segmentation trees, e.g. Ours $(\mathrm{ts}+\mathrm{bc})$ and Ours(ts+hc), outperform methods labeling on flat segmentations. In [37], they considered hierarchical segmentation by packing all semantic features together in a tree path. However, they still label on the flat leaf node segmentations, losing some visual information.

- Prediction becomes easier when classes are more general. Thus, for tasks where specificity is not strictly required, we can predict more general labels to achieve higher accuracy.

To further evaluate the labeling performance using our semantic hierarchy, we plot average information gain vs. accuracy curves (Fig. 5-left) and average class recall vs. accuracy curves (Fig. 5-right) by varying the degree of specificity for prediction parameter $\eta$. We compare approaches [37], [14]+[37] and our approaches using single ucm tree (Ours(ucm)), single normal tree (Ours(normal)), single semantic tree (Ours(category)) and using all three trees (Ours(full)). For the flat labeling approach [37], [14]+[37], we treat each class in the hierarchy as an arbitrary class without considering the hierarchy and train a one-vs-all SVM as in [37]. From these results, we can see that our approaches outperform the flat labeling approaches by a large margin, since the semantic hierarchy is considered. For the same de- 


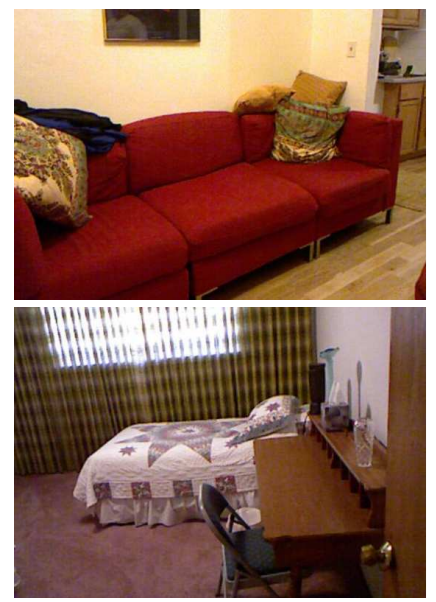

(a) Input Image

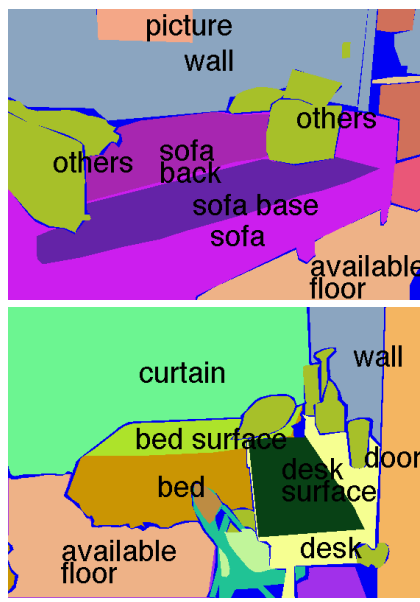

(b) Ground Truth

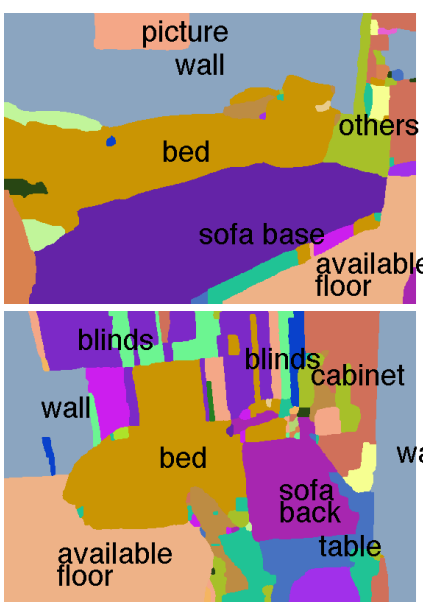

(c) $14+37$

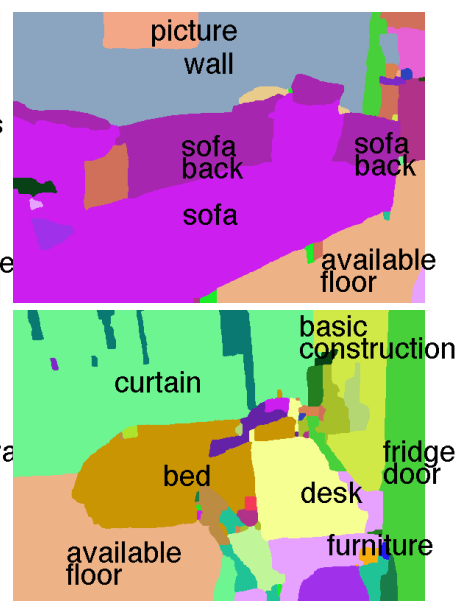

(d) Ours

Fig. 6: Some samples of the results on NYD2 dataset (small areas are not shown with label names for clarity). In the first row, sofa back is labeled correctly since semantic hierarchy (sofa,sofa back) is considered. In the second row, our algorithm labeled the higher level classes desk, basic construction instead of desk surface, wall to avoid possible mistakes with the help of semantic hierarchy.
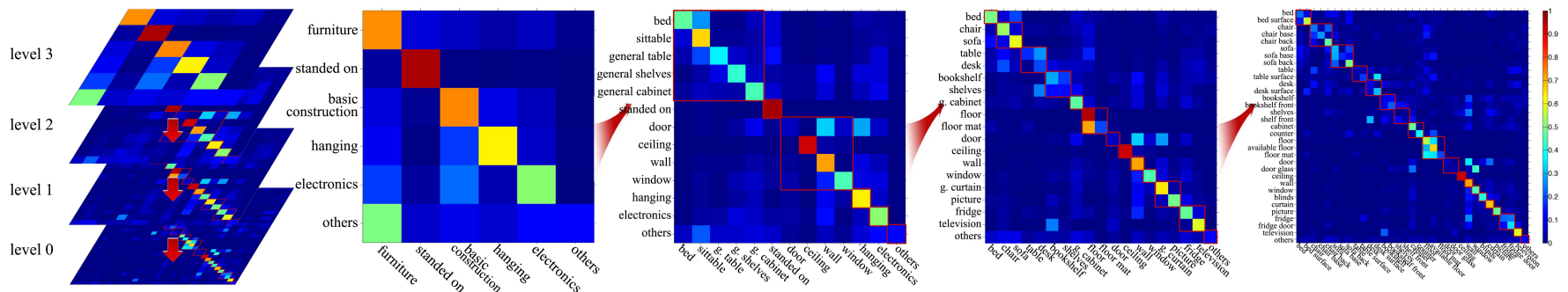

Fig. 7: Multi-level confusion matrix of our final results on NYUD2 dataset. From left to right, the confusion matrix zooms in to see more specific results in the next level below. In each confusion matrix, the red border square gives the classes merged in the next level up.

gree of specificity, our algorithms give higher accuracy. Using multiple segmentation trees also improves the performance.

We give two visual examples of labeling results in Fig. 6. In the first example, we can see that our algorithm yields a better labeling because semantic hierarchical relations such as (sofa,sofa back) are considered. The second example shows that the hierarchical labeling can use higher level classes to avoid possible mistakes, such as using desk or basic construction rather than desk surface or wall.

To further study the labeling results of our algorithm, we illustrate a multi-level confusion matrix in Fig. 7. We can see that some between-class labeling errors occur within one general class such as sofa, chair, stood on, most of which vanish in the next-higher level. However, some classes are hard to discriminate at any levels, such as door and wall, door and hanging. Our algorithm performed poorly for the background class others as it contains large variations in visual appearance.

\section{ROBOTIC EXPERIMENTS}

We evaluated our approach on three robotic tasks: object search, retrieval, and placement. We used a PR2 robot equipped with a Microsoft Kinect as our robotic platform. Table $\Pi$ s shows a summary of the results, listing the perception accuracy ('perc') and end-to-end execution ('exec') separately.

\section{A. Object Search Experiments}

Here the goal for the robot is to locate a particular object in a room by moving around 5 We compare our approach to [37]. For repeatable experiments, we pre-recorded a search tree at 20 discrete locations, each with a corresponding RGB-D frame (not in the training set).

We ran four separate trials for each algorithm, with the goal of searching for a chair back, fridge handle, mug handle, and baseball. To evaluate performance, the robot to takes a fixed number of steps, and then reports the location at which it had the highest confidence of finding the given object. We score the algorithm's performance based on the overlap ratio of the reported and ground-truth pixels of the target class for that frame, i.e. $\left|p_{d} \cap p_{g}\right| /\left|p_{d} \cup p_{g}\right|$, where $p_{d}, p_{g}$ are the detected object pixels and ground-truth object pixels.

Fig. 10 shows that for any fixed number of steps, our algorithm was able to outperform the approach from [37] for this task. Our algorithm was able to achieve an average overlap ratio of 0.4 after only 6 steps, while [37] took 15, showing that our approach does a better job of informing the search.

${ }^{5}$ Experimental setup details: The robot moves in discrete steps through the room, effectively moving through a search tree spanning the room. At each node in the tree, it turns to face each potential next location to move to, recording and labeling an RGB-D image for each. The robot will then move to the next location with the highest confidence score for containing the target object. If there are no unvisited neighboring locations, or this score is below some threshold, the robot will instead backtrack. 

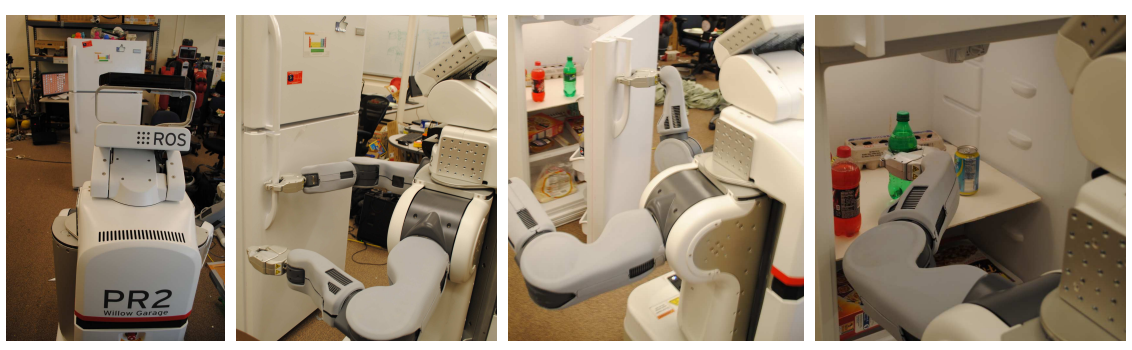

Fig. 8: Fetching a drink with our robot. A few snapshots of our algorithm running on our PR2 robot for the task of fetching a drink. From left to right: the robot starts some distance from the fridge, navigates to it using our labeling, detects the handle, and grasps it. It then opens the fridge, and finally retrieves a soda from it.

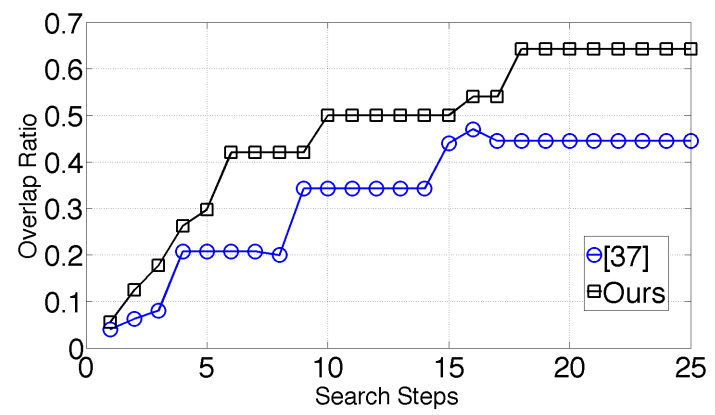

Fig. 10: Robot Object Search results. Figure shows the accuracy vs the number of movement steps taken by the robot.

TABLE II: Robotic experiment results. Success rates for perception ('perch') and actual robotic execution ('exec') of each task.

\begin{tabular}{|c|c|c|c|c|c|c|c|}
\hline & \multicolumn{2}{|c|}{ Search } & \multicolumn{3}{|c|}{ Retrieval } & \multirow{2}{*}{$\begin{array}{c}\text { Placement } \\
\text { Cushion } \\
\text { perc exec }\end{array}$} & \multirow{2}{*}{$\begin{array}{l}\text { Average } \\
\text { perc exec }\end{array}$} \\
\hline & $\begin{array}{l}\text { @ 20 } \\
\text { perc }\end{array}$ & $\begin{array}{l}\text { steps } \\
\text { exec }\end{array}$ & & $\begin{array}{l}\text { da } \\
\text { exec }\end{array}$ & $\begin{array}{c}\text { Bowl } \\
\text { perc exec }\end{array}$ & & \\
\hline & 44 & 44 & 33 & 33 & $38 \quad 38$ & $50 \quad 50$ & 42 \\
\hline Hiera & 64 & 64 & 90 & 80 & 80 & 100 & 84 \\
\hline
\end{tabular}

After 20 steps, both algorithms converged, and ours achieves an average overlap ratio of 0.64 versus the 0.44 ratio from the baseline approach, thus also improving long-term accuracy.

\section{B. Object Retrieval Experiments}

In this experiment, the robot has to perform a series of perception and motion/manipulation steps for retrieving an object - to fetch a drink from a fridge, and to fetch a bowl from a kitchen counter. The robot first detects and navigates to a semantically appropriate area to find the object in, then locates the target object, grasps it, and brings it back.

In some cases, the desired object may not be available, and the robot is then allowed to retrieve an appropriate substitute. We define this as some other descendant of a class's parent in the semantic hierarchy - for example, Pepsi is a substitute for Coke because both have the parent class soda. A flat labeling scheme is incapable of determining such substitutes, and will report failure if the target class is not found.

From Table II we can see that our algorithm achieves a very high rate of success for the complex drink-retrieval task shown in Fig. 8 Even though this task requires three separate phases of perception, our perception algorithm failed only once in ten trials, failing to find the fridge handle, giving a 90\% perception success rate. One more execution failure was due to the fridge door swinging closed before

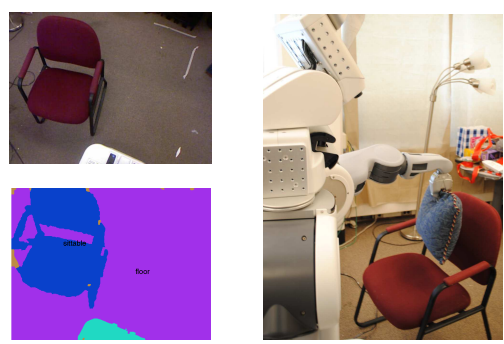

Fig. 9: Placing a cushion. No sofa was present, but the robot used our hierarchy to determine that the chair was another sittable object and thus a reasonable place for the cushion.

the robot could hold it open, giving an $80 \%$ overall success rate. Results for the bowl retrieval experiment were similar. Video of some of these experiments is available at: http: //pr.cs.cornell.edu/sceneunderstanding/.

At long distances, neither ours nor the baseline labeling algorithms were able to distinguish the handle from the door of the fridge, but our hierarchy informed the robot that the handle was part of the door. The flat labeling approach, meanwhile, lacked this information and simply failed if it could not identify the handle. In fact, the robot was only able to open the fridge $50 \%$ of the times using flat labels. Once opened, it could not identify proper substitutes if the desired drink was not present, leading to a mere $33 \%$ perception success rate.

\section{Object Placement Experiments}

We also performed a series of experiments in which the robot's goal was object placement rather than retrieval. In particular, we considered the task of placing a cushion on a sofa, or on some other sittable object such as a chair if a sofa is not present. In every experiment performed, our algorithm was able to successfully locate the sofa, or a substitute if there was no sofa. One example of the robot successfully placing a cushion is shown in Fig. 9. By contrast, when using a flat labeling approach, the robot did not understand to place the cushion on another sittable surface if the sofa was not present, and thus succeeded only in the $50 \%$ of cases.

\section{CONCLUSION}

Objects in human environments can be classified into a meaningful hierarchy, both because these objects are composed of parts (e.g. fridge-fridge door-fridge handle) and because of different levels of abstraction (e.g. drink-sodaCoke). Modeling this is very important in enabling a robot to perform many tasks in these environments. In this work, we developed an approach to labeling a segmentation tree with such hierarchical semantic labels. We presented a model based on a Conditional Random Field which incorporated several constraints to allow labeling using this hierarchy. Our model allows for different levels of specificity in labeling, while still remaining tractable for inference. We showed that our method outperforms state-of-the-art scene labeling approaches on a standard dataset (NYUD2), and demonstrated its use on several robotic tasks. 


\section{REFERENCES}

[1] Mixed integer programming solver. http://tomopt.com/tomlab/ products/cplex/

[2] E. A. Akkoyunlu. The enumeration of maximal cliques of large graphs. SIAM J. Comput., 2(1):1-6, 1973.

[3] D. R. Aloysius. Bron-Kerbosch Algorithm. PopulPublishing, 2012. ISBN 6136404559, 9786136404554.

[4] A. Anand, H. S. Koppula, T. Joachims, and A. Saxena. Contextually guided semantic labeling and search for three-dimensional point clouds. IJRR, 32(1):19-34, 2013.

[5] P. Arbelaez, M. Maire, C. Fowlkes, and J. Malik. Contour detection and hierarchical image segmentation. PAMI, 33(5): 898-916, 2011.

[6] A. Aydemir, K. Sjöö, J. Folkesson, A. Pronobis, and P. Jensfelt. Search in the real world: Active visual object search based on spatial relations. In ICRA, 2011.

[7] C. Bron and J. Kerbosch. Algorithm 457: Finding all cliques of an undirected graph. Commun. ACM, 16(9):575-577, 1973.

[8] S. X. Chen, A. Jain, A. Gupta, and L. S. Davis. Piecing together the segmentation jigsaw using context. In $C V P R, 2011$.

[9] J. Deng, W. Dong, R. Socher, L.-J. Li, K. Li, and L. Fei-Fei. Imagenet: A large-scale hierarchical image database. In $C V P R$, 2009.

[10] J. Deng, J. Krause, A. Berg, and L. Fei-Fei. Hedging your bets: Optimizing accuracy-specificity trade-offs in large scale visual recognition. In $C V P R, 2012$.

[11] C. Eppner and O. Brock. Grasping unknown objects by exploiting shape adaptability and environmental constraints. In IROS, 2013.

[12] S. Gould, R. Fulton, and D. Koller. Decomposing a scene into geometric and semantically consistent regions. In ICCV, 2009.

[13] K. Granström, J. Callmer, F. T. Ramos, and J. I. Nieto. Learning to detect loop closure from range data. In ICRA, 2009.

[14] S. Gupta, P. Arbelaez, and J. Malik. Perceptual organization and recognition of indoor scenes from RGB-D images. In $C V P R$, 2013.

[15] H. He and B. Upcroft. Nonparametric semantic segmentation for 3d street scenes. In IROS, 2013.

[16] E. Herbst, X. Ren, and D. Fox. RGB-D flow: Dense 3-d motion estimation using color and depth. In ICRA, 2013.

[17] E. Herbst, P. Henry, and D. Fox. Toward online 3-d object segmentation and mapping. In ICRA, 2014.

[18] L. Hinkle and Edwin. Predicting object functionality using physical simulations. In IROS, 2013.

[19] Z. Jia, A. Gallagher, A. Saxena, and T. Chen. 3d-based reasoning with blocks, support, and stability. In CVPR, 2013.

[20] Y. Jiang, H. Koppula, and A. Saxena. Hallucinated humans as the hidden context for labeling 3d scenes. In CVPR, 2013.

[21] M. Kaess, H. Johannsson, R. Roberts, V. Ila, J. J. Leonard, and F. Dellaert. iSAM2: Incremental smoothing and mapping using the bayes tree. IJRR, 31(2):216-235, 2012.

[22] D. Katz and O. Brock. Interactive segmentation of articulated objects in 3d. In Workshop on Mobile Manipulation at ICRA, 2011.

[23] H. Koppula and A. Saxena. Anticipating human activities using object affordances for reactive robotic response. In RSS, 2013.

[24] H. Koppula, A. Anand, T. Joachims, and A. Saxena. Semantic labeling of 3d point clouds for indoor scenes. In NIPS, 2011.

[25] H. Koppula, R. Gupta, and A. Saxena. Learning human activities and object affordances from rgb-d videos. IJRR, 32 (8):951-970, 2013.

[26] D. Kottas and S. Roumeliotis. Exploiting urban scenes for vision-aided inertial navigation. In RSS, 2013.

[27] K. Lai, L. Bo, and D. Fox. Unsupervised feature learning for 3d scene labeling. In ICRA, 2014.

[28] M. T. Lazaro, L. M. Paz, P. Pinies, J. A. Castellanos, and
G. Grisetti. Multi-robot SLAM using condensed measurements. In IROS, 2013.

[29] I. Lenz, H. Lee, and A. Saxena. Deep learning for detecting robotic grasps. In RSS, 2013.

[30] M. Lorbach, S. Hfer, and O. Brock. Prior-assisted propagation of spatial information for object search. In IROS, 2014.

[31] M. Madry, C. H. Ek, R. Detry, K. Hang, and D. Kragic. Improving generalization for $3 \mathrm{~d}$ object categorization with global structure histograms. In IROS, 2012.

[32] T. Malisiewicz and A. A. Efros. Improving spatial support for objects via multiple segmentations. In $B M V C, 2007$.

[33] M. Milford. Vision-based place recognition: how low can you go? IJRR, 32(7):766-789, 2013.

[34] J. Neira, A. Davison, and J. Leonard. Guest editorial special issue on visual SLAM. Robotics, IEEE Transactions on, 24(5): 929-931, 2008.

[35] V. Ordonez, J. Deng, Y. Choi, A. C. Berg, and T. L. Berg. From large scale image categorization to entry-level categories. In $I C C V, 2013$.

[36] D. Pangercic, M. Tenorth, B. Pitzer, and M. Beetz. Semantic object maps for robotic housework - representation, acquisition and use. In IROS, 2012.

[37] X. Ren, L. Bo, and D. Fox. RGB-D scene labeling: Features and algorithms. In CVPR, 2012.

[38] M. Ruhnke, L. Bo, D. Fox, and W. Burgard. Compact RGB-D surface models based on sparse coding. In AAAI, 2013.

[39] B. C. Russell and A. Torralba. Building a database of $3 \mathrm{~d}$ scenes from user annotations. In $C V P R, 2009$.

[40] R. F. Salas-Moreno, R. A. Newcombe, H. Strasdat, P. H. J. Kelly, and A. J. Davison. SLAM++: Simultaneous localisation and mapping at the level of objects. In CVPR, 2013.

[41] A. Saxena, S. Chung, and A. Ng. Learning depth from single monocular images. In NIPS, 2005.

[42] A. Saxena, J. Driemeyer, and A. Ng. Robotic grasping of novel objects using vision. IJRR, 27(2):157, 2008.

[43] A. Saxena, M. Sun, and A. Ng. Make3d: Learning 3d scene structure from a single still image. PAMI, 31(5):824-840, 2009.

[44] S. Shen, Y. Mulgaonkar, N. Michael, and V. Kumar. Visionbased state estimation and trajectory control towards high-speed flight with a quadrotor. In RSS, 2013.

[45] N. Silberman, D. Hoiem, P. Kohli, and R. Fergus. Indoor segmentation and support inference from RGB-D images. In ECCV, 2012.

[46] T. Whelan, H. Johannsson, M. Kaess, J. Leonard, and J. McDonald. Robust real-time visual odometry for dense RGB-D mapping. In ICRA, 2013. 